\title{
Estudios de diario en la investigación sobre emociones, empleo, desempleo y género: estado de la cuestión
}

\author{
ÚRSULA NEBOT PRAT \\ al001834@uji.es \\ YAZMin MONTEAGUdo CÁCERES \\ al295809@uji.es \\ Eva Cifre Gallego \\ cifre@uji.es
}

\section{Resumen}

Introducción: El creciente interés por la investigación de los procesos que ocurren en el corto plazo y en contextos naturales ha propiciado un incremento de los estudios de diario en áreas como salud y estrés. En esta línea, dentro del proyecto «Calidad de vida en jóvenes: importancia de la regulación emocional en empleados y desempleados», contemplamos la realización de un estudio de diario. Objetivo: Revisar la bibliografía publicada sobre metodología de estudios de diario y su aplicación en diferentes contextos. Metodología: Búsqueda de información en bases de datos científicas del ámbito de la psicología a través de palabras clave relacionadas con nuestra temática de investigación. Resultados: Se ha elaborado una tabla resumen de publicaciones que incluye preguntas de investigación, muestra, procedimiento, variables, medidas, formato, análisis y resultados. Las ventajas más recalcadas de los estudios de diario han sido el estudio en contextos naturales y la reducción de los errores de la retrospección. Las investigaciones que utilizan estudios de diario en el campo del desempleo son más reducidas que en el ámbito laboral, y más aún si se tiene en cuenta la variable género. Los estudios suelen estar basados en eventos o experiencias y se centran en obtener los datos al final del día o varias veces al día. Discusión: Este estudio nos ha permitido extraer las ideas principales de lo que es un estudio de diario, así como resaltar la importancia de diseñar estos estudios para dar respuesta a las preguntas de investigación.

Palabras clave: estudio de diario, emociones, empleo, desempleo, género.

\section{Abstract}

Introduction: The growing interest in investigating processes that occur in the short term and in natural contexts has led to increasingly more diary studies in areas like health and stress. In line with this, the project «Quality of Life in Youth: Importance of Emotional Regulation in Employed and Unemployed People» includes a diary study. Aim: To review the published literature on the diary study methodology and its daily application in 
different contexts. Methods: We searched for information in scientific databases in the psychology field using keywords related to our research topic. Results: We developed a summary table of publications that includes: research questions, samples, procedures, variables, dimensions, formats, analyses and Results: The most stressed advantages of diary studies are that that they are conducted in natural contexts and they reduce retrospection errors. Fewer research works have used diary studies in the unemployment field than in the workplace, and fewer still exist when we consider the gender variable. Studies are often based on events or experiences, and focus on collecting data at the end of the day or several times a day. Discussion: This study allowed us to draw the main ideas of what a diary study is, and to highlight the importance of designing studies that centre on answering research questions.

Keywords: diary study, emotions, work, unemployment, gender.

\section{Introducción}

En el campo de la psicología del trabajo y las organizaciones existe un creciente interés por la investigación de los procesos a corto plazo y de las experiencias diarias de las personas. La experiencia diaria sugiere que no siempre tenemos el mismo estado de ánimo y que el desempeño laboral puede fluctuar de día a día. Los estudios de diario proporcionan los medios necesarios para examinar estos procesos (Ohly, Sonnentag, Niessen y Zapf, 2010).

En un estudio de diario las personas proporcionan informes frecuentes de los eventos y experiencias de su vida diaria, permitiendo capturar las particularidades de estas experiencias diarias de una forma que no es posible usando diseños tradicionales (Bolger, Davis y Rafaeli, 2003). Frente a los diseños transversales, ampliamente utilizados en la investigación y cuyo foco está en las diferencias entre las personas (interpersona), el estudio de diario longitudinal permite capturar las dinámicas y fluctuaciones a corto plazo y efectuar diferentes análisis considerando la misma persona (intrapersona).

Con otras palabras, los estudios de diario permiten capturar la vida tal y como es vivida (Bolger y cols., 2003). Posibilitan el estudio de los pensamientos, sentimientos y conductas en el contexto natural de trabajo. $Y$ todo ello ha propiciado su incremento particularmente en las áreas de salud y estrés, emociones y trabajo, relación trabajo-hogar e interacciones sociales (Ohly y cols., 2010).

En esta dirección, dentro del proyecto «Calidad de vida en jóvenes: importancia de la regulación emocional en empleados y desempleados», desarrollado por el grupo de investigación MPAGERde la Universitat Jaume I, se contempla la realización de un estudio de diario. El objetivo general de dicho proyecto es poner a prueba el papel moderador de la regulación emocional sobre las emociones negativas provocadas por la situación de desempleo o de inseguridad laboral de los jóvenes (en particular) y las emociones de dichos jóvenes y las consecuencias asociadas (en general), como la calidad de vida y el capital psicológico positivo. El proyecto consta de varios estudios específicos que incluyen tanto metodología cualitativa como cuantitativa, con diseños transversales y longitudinales con estudio de diario, el cual permitirá establecer una causalidad entre las variables de estudio. Es en esta última fase de estudio de diario donde se inserta el presente trabajo, el cual tiene como objetivo revisar la bibliografía publicada sobre la metodología de estudios de diario y su aplicación en diferentes contextos, incluyendo desempleo y género. 


\section{Método}

En una primera fase, para una aproximación a la metodología de estudios de diario, se ha partido de la lectura de publicaciones básicas introductorias del tema, extrayendo las ideas principales. Aunque los estudios de diario han empezado a ser más populares en los últimos años, las investigadoras e investigadores no están familiarizados con estos y encuentran dificultades para acceder a la información requerida (Ohly y cols., 2010). Existen diversas revisiones que sirven de introducción a la metodología de estudios de diario, entre los que caben destacar los de Bolger y cols. (2003), Ohly y cols. (2010) y Reis y Gable ( 2000), donde se contemplan los diferentes tipos de estudios de diario, las preguntas de investigación que se pueden examinar, el tamaño de la muestra, la tecnología utilizada para la obtención de los informes diarios o las estrategias de análisis de datos a través de modelos multinivel.

En una segunda fase, para detectar las investigaciones que utilizan estudios de diario, se ha recurido a la búsqueda de información en las bases de datos científicas del ámbito de la psicología Psyarticles, PubPsych y PsycNet, incluyendo palabras clave relacionadas con nuestra temática de investigación: diary study, emotion, work, unemployment y gender.

En una tercera fase, se ha realizado una selección y revisión en profundidad de las investigaciones con estudios de diario publicadas, por temática, autoría y año que contemplan las variables emociones, desempleo y género. Se ha elaborado una tabla resumen con las diversas preguntas de investigación abordadas, el metódo de investigación utilizado, el formato de los cuestionarios, el tamaño y las características de la muestra, las variables de estudio, las medidas utilizadas y los análisis y resultados más significativos. No se ha realizado el análisis de investigaciones sobre empleo o contextos de trabajo al haber ya publicados otros artículos que realizan una revisión de los estudios de diario en el ámbito del trabajo y que muestran tablas resumen similares a la anteriormente indicada (Ohly y cols., 2010).

\section{Resultados}

En la primera fase, con la revisión bibliográfica se han extraído las características más relevantes de los estudios de diario, que se presentan a continuación.

Los estudios de diario utilizan cuestionarios estructurados con preguntas estandarizadas y una gran parte están basados en el muestreo de eventos o en el muestreo de experiencias. En el muestreo de eventos quien participa tiene que informar de un evento, por ejemplo una interacción con responsables, colegas o clientes, un conflicto o un evento estresante. La cuestión es limitar el número de eventos a informar y darle a quien participa una guía de los eventos de los que puede informar. El muestreo de experiencias implica el recuerdo in situ de los pensamientos, los sentimientos y las conductas, las señales y sus consecuencias. Con un ordenador portátil, por ejemplo, se puede dar una señal de aviso de forma aleatoria o en puntos fijos en el tiempo y quienes participan tienen que contestar de forma inmediata el cuestionario. Se ha usado por ejemplo para evaluar la frecuencia y el contexto de los estados de flow. En otras ocasiones, se evalúan las experiencias y procesos ocurridos durante el día sin hacer referencia a un evento en particular. Por ejemplo, la experiencia diaria de trabajo cambia y es evaluada por la tarde. En comparación con el muestreo de experiencias, aquí las respuestas se retrasan y los diarios son completados normalmente al final del día (Ohly y cols., 2010).

Interesa analizar si las variables de interés fluctúan de la mañana a la noche, si se comportan de manera diferente los fines de semana y los días entre semana o si tienen un cierto crecimiento durante varias semanas (Bolger y cols., 2003). Para ello, los cuestionarios se cumplimentan al final del día o en varios momentos del día, durante, por ejemplo, cinco días consecutivos entre semana o durante varias semanas. 
Los estudios de diario tienen algunas ventajas en comparación con otros diseños de investigación. En primer lugar, permiten el examen de los eventos y experiencias en su contexto natural. En segundo lugar, reducen los errores de la retrospección (Reis y Gable, 2000), minimizando la cantidad de tiempo transcurrido entre una experiencia y el relato de esta experiencia. Una de las limitaciones que presentan los estudios de diario es el alto nivel de compromiso y dedicación de las personas que participan en el estudio, por las múltiples evaluaciones que se solicitan, siendo de gran importancia los mecanismos de reclutamiento y motivación de la muestra que se utilicen. Se recomienda que las evaluaciones diarias no excedan de 5-7 minutos (Reis y Gable, 2000), con escalas abreviadas y adaptadas, monoítems e ítems seleccionados con la mayor puntuación total en la correlación de escalas de ítems múltiples. Además, los estudios de diario a menudo requieren detalladas sesiones de formación para garantizar que quienes participan comprenden plenamente el protocolo.

Mayoritariamente se realizan estudios de diario con cuestionarios en formato de lápiz y papel, aunque en los últimos años se ha ido introduciendo el uso de las nuevas tecnologías como cuestionarios online o telefonía móvil, lo que está facilitando tanto la recogida de datos como su posterior análisis.

En la segunda fase, la búsqueda de información en bases de datos científicas realizada a través de operadores booleanos y palabras clave ha arrojado los primeros resultados que se muestran en la tabla 1.

Al observar los datos, se confirma el interés en los estudios de diario aplicados a la investigación del trabajo, a la vez que se detecta que en el campo del desempleo son muy reducidos. Respecto al estudio de las emociones y el género, vemos que las emociones aparecen como objeto de estudio destacado, pero la consideración del género como variable de estudio es limitada.

Tabla 1

Número de artículos encontrados por base de datos y palabras clave

\begin{tabular}{|c|c|c|c|}
\hline \multirow[b]{2}{*}{ Palabras clave } & \multicolumn{3}{|c|}{ Bases de datos } \\
\hline & PsycNet & PubPsych & PsyARTICLES \\
\hline "Diary Method" OR "Diary Study" OR “Daily Diary" & 1699 & 458 & 253 \\
\hline $\begin{array}{l}\text { ("Diary Method" OR "Diary Study" OR "Daily Diary") } \\
\text { AND (Employ* OR Work OR Job) }\end{array}$ & 395 & 153 & 69 \\
\hline $\begin{array}{l}\text { ("Diary Method" OR "Diary Study" OR "Daily Diary") } \\
\text { AND Unemploy* }\end{array}$ & 2 & 0 & 0 \\
\hline $\begin{array}{l}\text { ("Diary Method" OR "Diary Study" OR "Daily Diary") aND } \\
\text { Emotion* }\end{array}$ & 551 & 154 & 111 \\
\hline $\begin{array}{l}\text { (“Diary Method” OR “Diary Study” OR “Daily Diary”) } \\
\text { AND Gender }\end{array}$ & 103 & 26 & 17 \\
\hline $\begin{array}{l}\text { ("Diary Method" OR "Diary Study" OR "Daily Diary") } \\
\text { AND Emotion* AND Gender }\end{array}$ & 33 & 16 & 5 \\
\hline $\begin{array}{l}\text { ("Diary Method" OR "Diary Study" OR "Daily Diary") } \\
\text { AND (Employ* OR Work OR Job) AND Emotion* }\end{array}$ & 128 & 48 & 25 \\
\hline $\begin{array}{l}\text { ("Diary Method" OR "Diary Study" OR "Daily Diary") } \\
\text { AND Unemploy* AND Emotion* }\end{array}$ & 1 & 0 & 0 \\
\hline $\begin{array}{l}\text { ("Diary Method" OR "Diary Study" OR "Daily Diary") } \\
\text { AND (Employ* OR Work OR Job) AND Gender }\end{array}$ & 27 & 4 & 4 \\
\hline
\end{tabular}




\begin{tabular}{|c|c|c|c|}
\hline \multirow[b]{2}{*}{ Palabras clave } & \multicolumn{3}{|c|}{ Bases de datos } \\
\hline & PsycNet & PubPsych & PsyARTICLES \\
\hline $\begin{array}{l}\text { ("Diary Method” OR “Diary Study" OR "Daily Diary”) } \\
\text { AND Unemploy* AND Gender }\end{array}$ & 0 & 0 & 0 \\
\hline $\begin{array}{l}\text { ("Diary Method" OR "Diary Study" OR "Daily Diary") aND } \\
\text { (Employ* OR Work OR Job) AND Emotion* AND Gender }\end{array}$ & 9 & 3 & 1 \\
\hline $\begin{array}{l}\text { (“Diary Method” OR "Diary Study" OR “Daily Diary”) } \\
\text { AND Unemploy* AND Emotion* AND Gender }\end{array}$ & 0 & 0 & 0 \\
\hline
\end{tabular}

En la tercera fase, del total de artículos encontrados (véase la tabla 1), diecinueve han sido analizados en profundidad. La tabla 2 presenta los resultados de una muestra representativa del total de los estudios de diario analizados. En el ámbito del desempleo y las emociones encontramos investigaciones que estudian la relación entre las experiencias diarias de búsqueda de empleo y los niveles de distress en la persona (Song, Uy, Zhang y Shi, 2009), cómo el estrés por desempleo afecta a nivel de pareja (Song, Foo, Uy y Sun, 2011)

o la relación entre desempleo, usos del tiempo y bienestar psicológico (Kilpatrick y Trew, 1985). Parte de los estudios evalúan la relación entre el funcionamiento emocional de las personas y diferentes estresores diarios como, por ejemplo, interacciones sociales y familiares negativas o eventos financieros negativos (Bolger, DeLongis, Kessler y Schilling, 1989; Genet y Siemer, 2012; Hankin, Fraley y Abela, 2005; Laurenceau y Bolger, 2005; Sturgeon, Zautra y Okun, 2014). La creatividad y las emociones (Conner y Silvia, 2015)

son otro ejemplo de estudios de diario. Otras publicaciones se centran en la relación entre género, como objeto principal de estudio, y emociones diarias (Aubé, 2008; Curran, McDaniel, Pollitt y Totenhagen, 2015; Witt y Wood, 2010)

\section{Discusión y conclusiones}

En el presente artículo se han revisado investigaciones previas basadas en estudios de diario y se han puesto de relieve diferentes cuestiones metodológicas que se deben abordar al llevar a cabo un estudio de este tipo. La investigación con estudios de diario nos brinda una oportunidad única de aproximarnos a los procesos intrapersonales que tienen lugar en el corto plazo. Para utilizar de manera efectiva los estudios de diario, debemos conocer sus ventajas y sus limitaciones. Así, el eje principal de todo estudio de diario tiene que partir de adecuar el diseño del estudio a las preguntas de investigación planteadas.

Con la metodología de estudios de diario se abre todo un abanico de posibilidades de investigación de los procesos psicológicos a corto plazo, estudiando pensamientos, sentimientos y comportamientos que se experimentan de forma diaria en su contexto natural. Y esto se está viendo ya reflejado en el incremento de las investigaciones que utilizan estudios de diario en el campo de la psicología del trabajo y las organizaciones. Sin embargo, para seguir contribuyendo al avance del conocimiento de los procesos a corto plazo, es necesario dar un paso más e incrementar los estudios de diario en áreas específicas como el desempleo y las emociones. Además, se vuelve cada vez más imprescindible incorporar el género en las investigaciones, tanto como variable de análisis como perspectiva transversal en toda investigación, tal y como marcan las políticas de investigación e innovación recientes (por ejemplo, en el actual Horizonte 2020). Este es un reto al que, desde la investigación, todavía tenemos que hacer frente. 


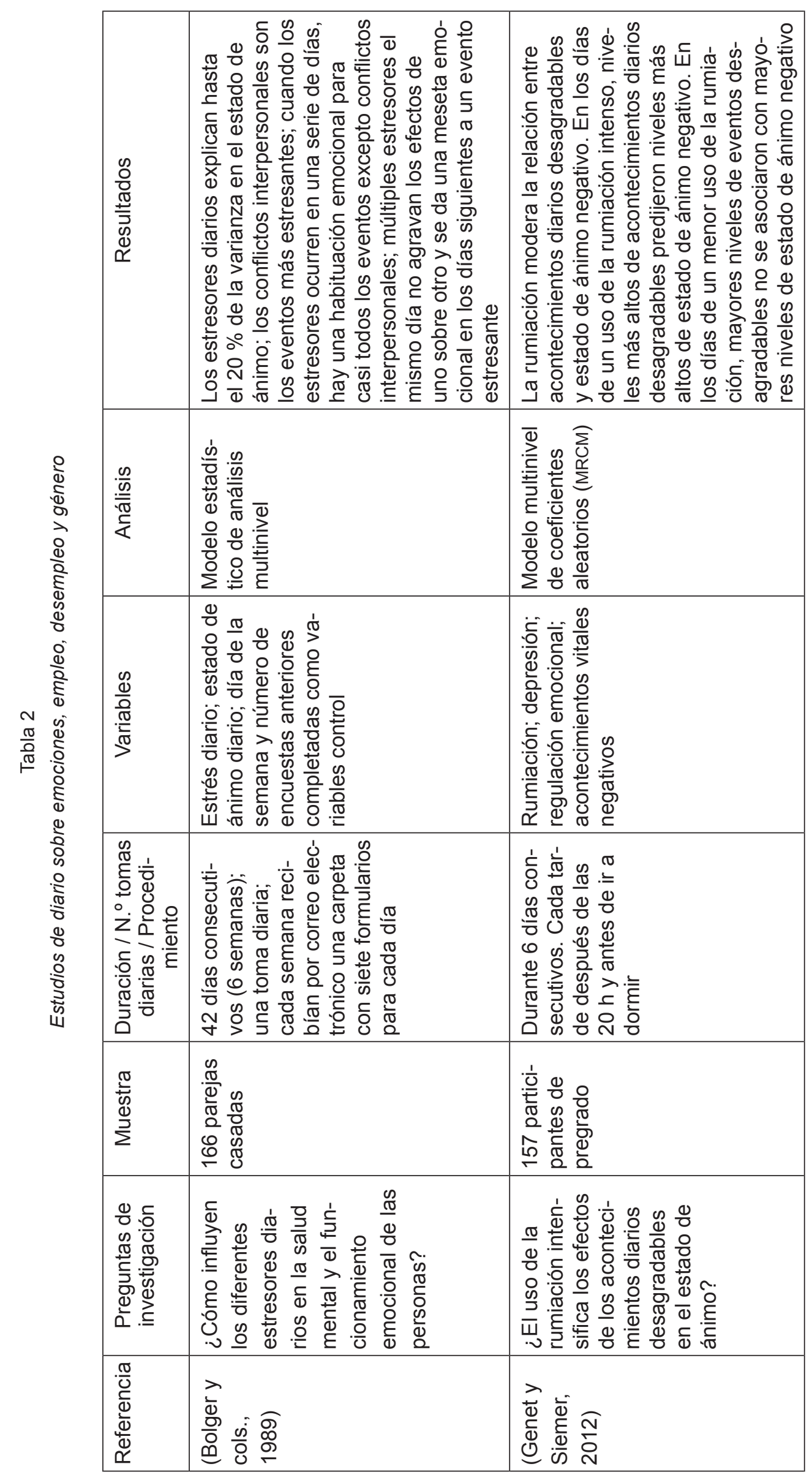




\begin{tabular}{|c|c|c|}
\hline 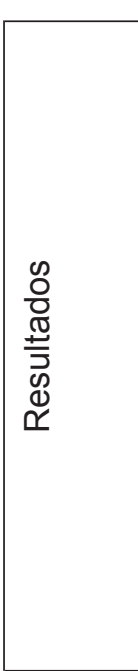 & 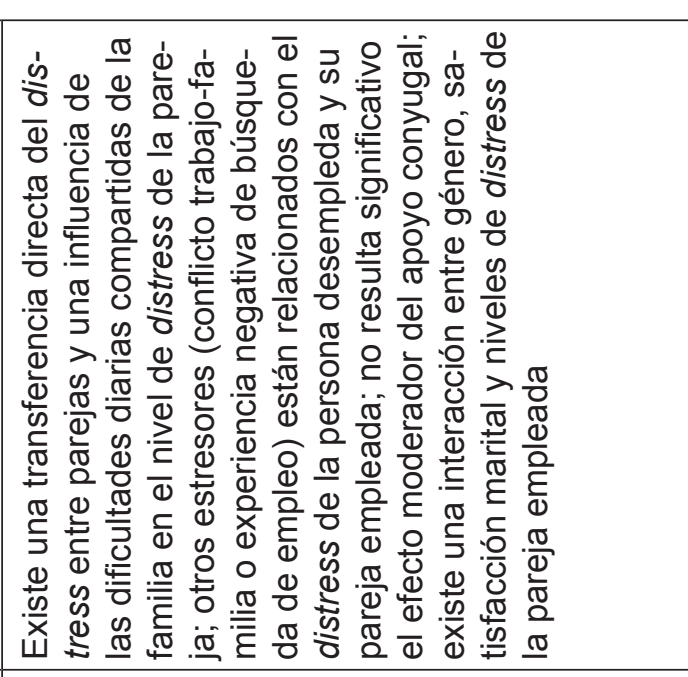 & 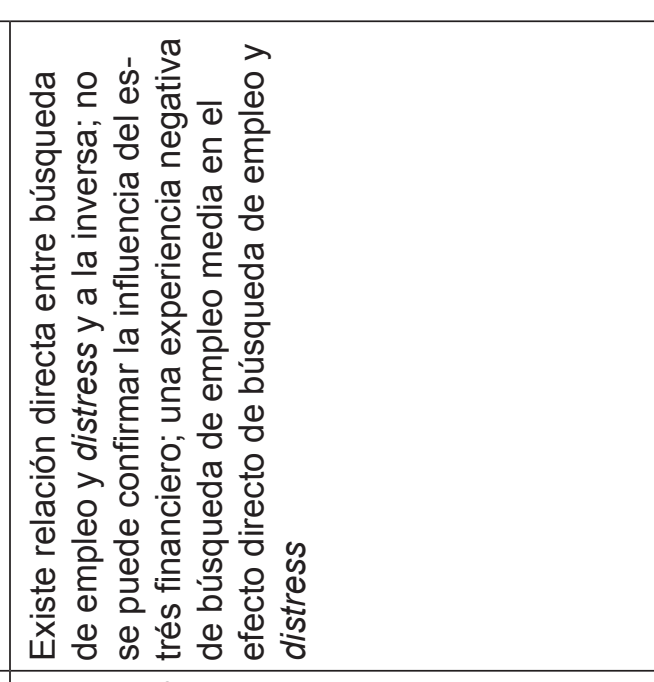 \\
\hline 员 & 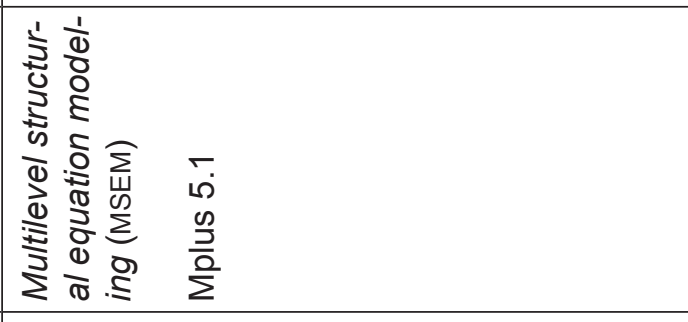 & 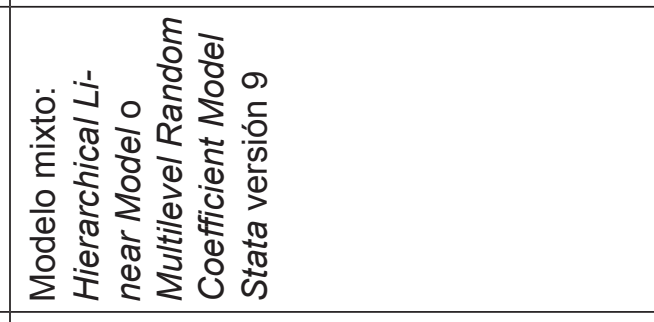 \\
\hline 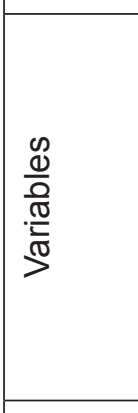 & 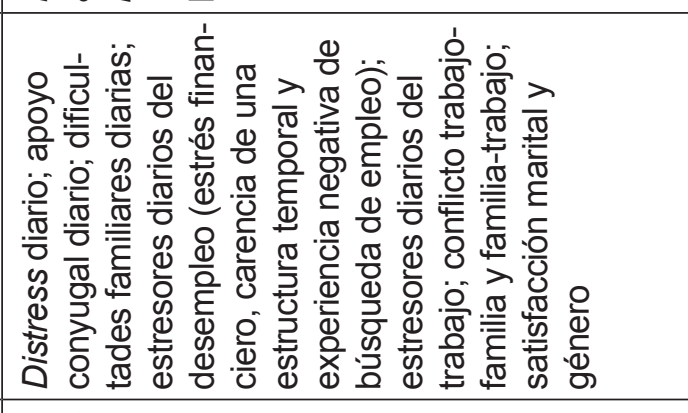 & 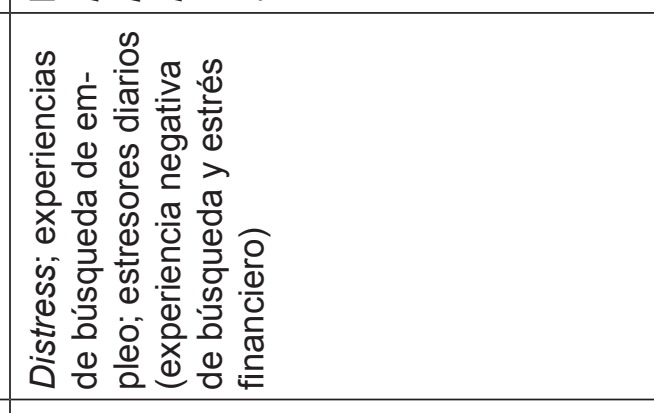 \\
\hline 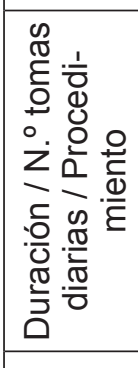 & 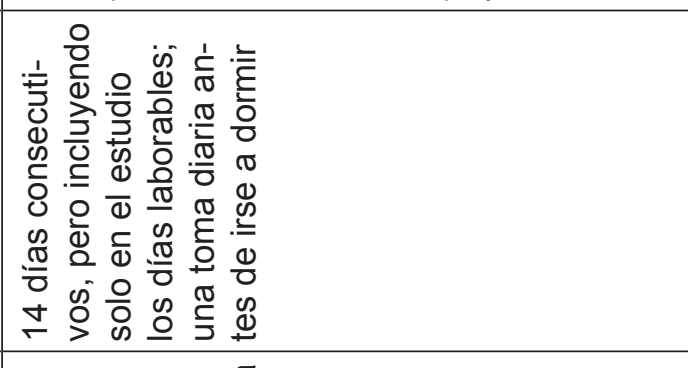 & 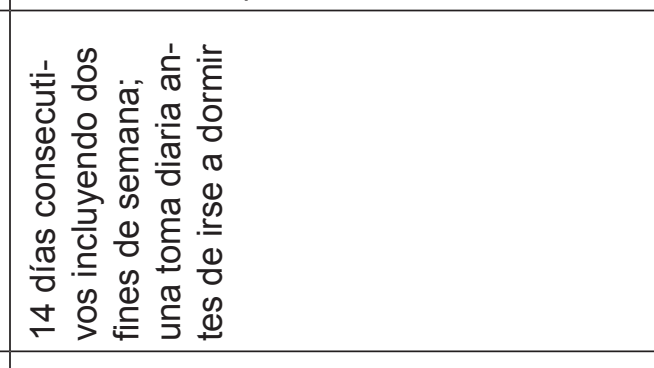 \\
\hline 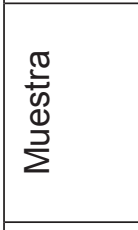 & 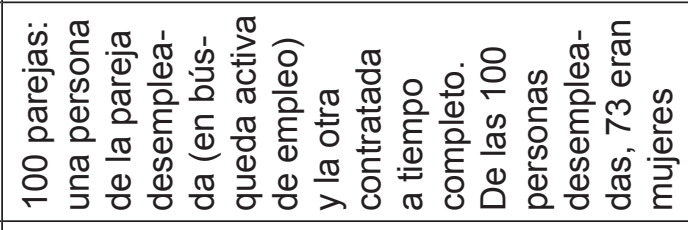 & 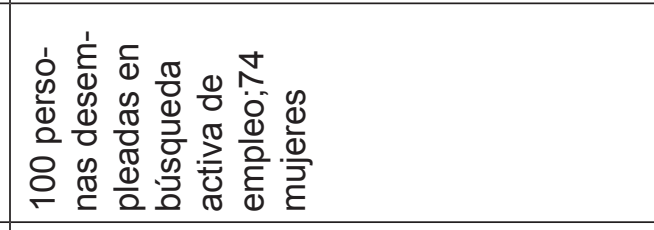 \\
\hline 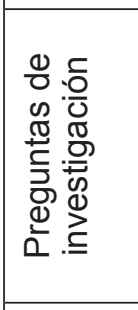 & 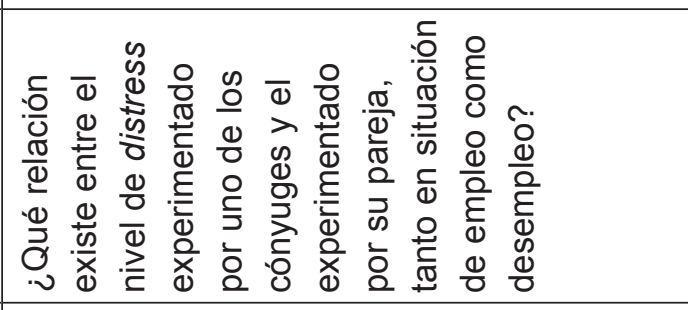 & 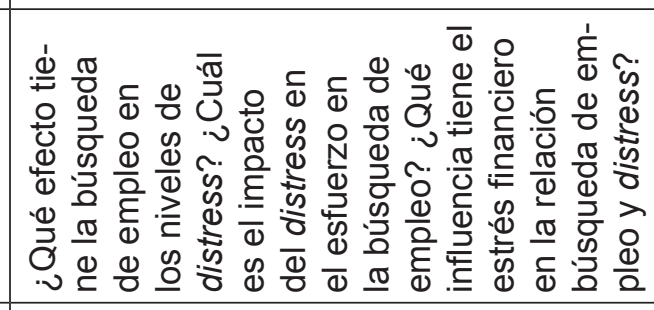 \\
\hline & 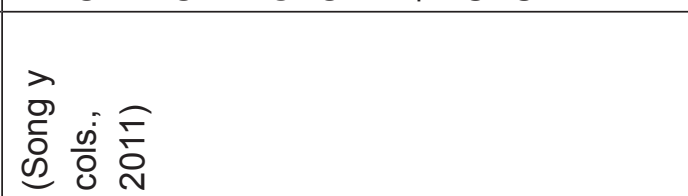 & 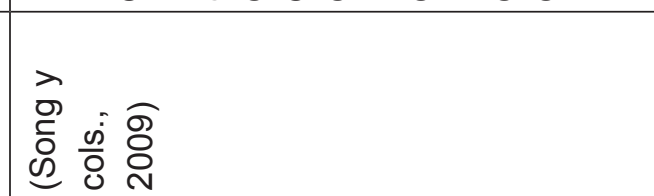 \\
\hline
\end{tabular}




\begin{tabular}{|c|c|c|}
\hline \multirow{7}{*}{ 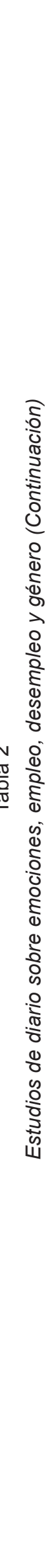 } & 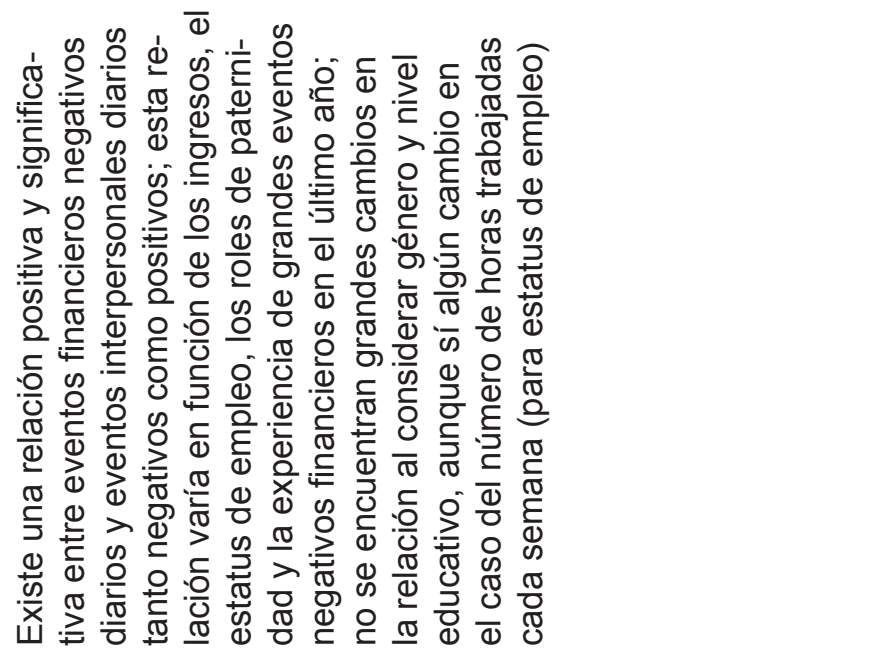 & 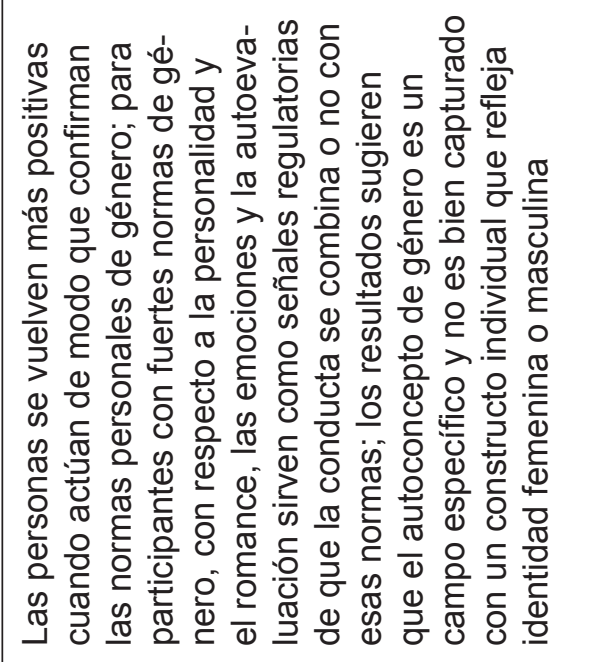 \\
\hline & 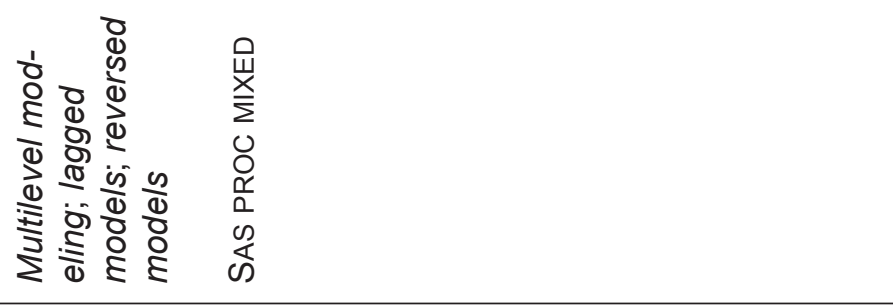 & 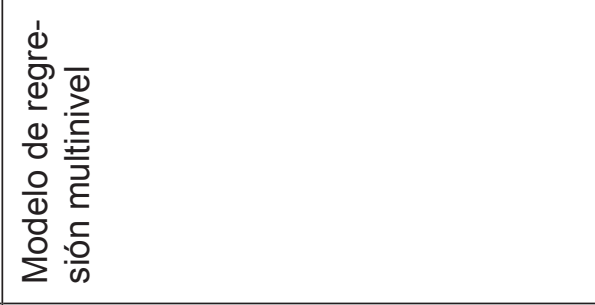 \\
\hline & 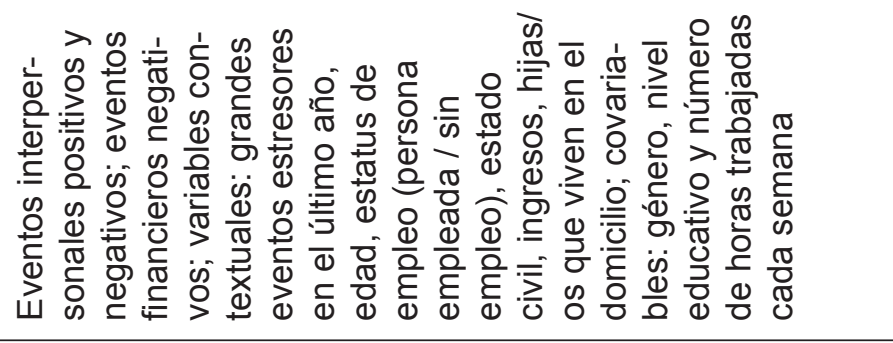 & 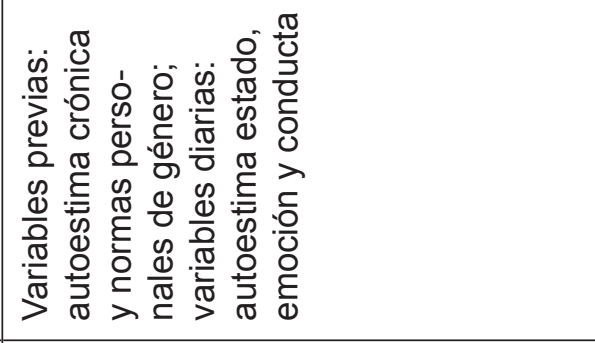 \\
\hline & 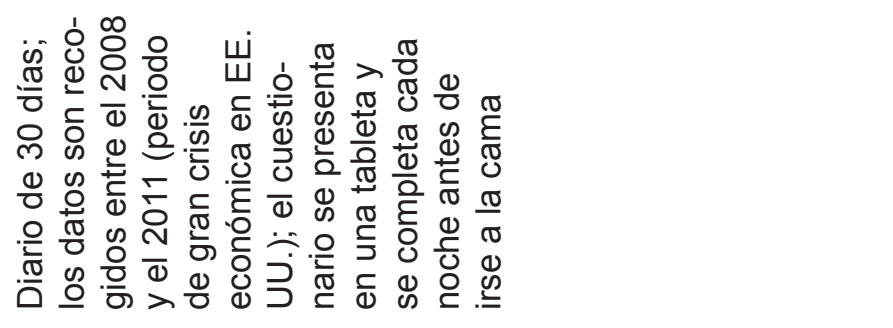 & 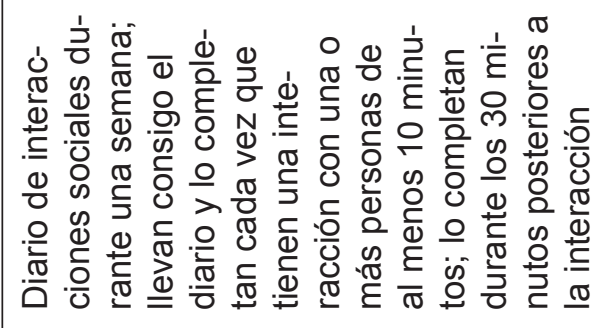 \\
\hline & 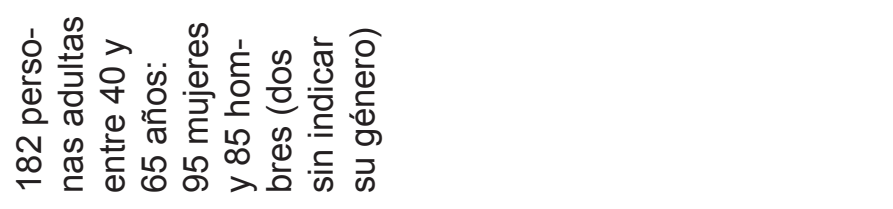 & 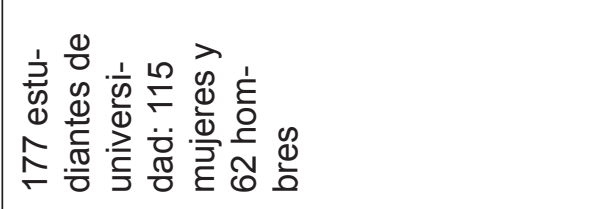 \\
\hline & 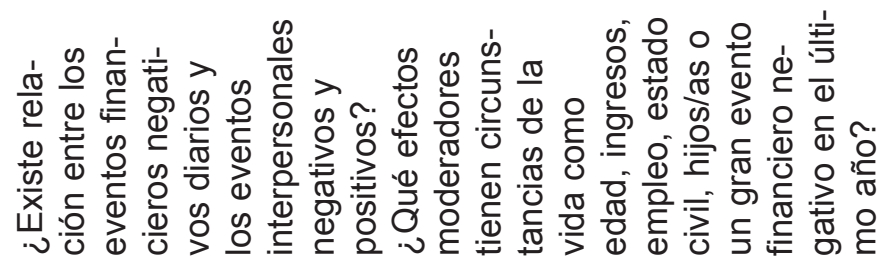 & 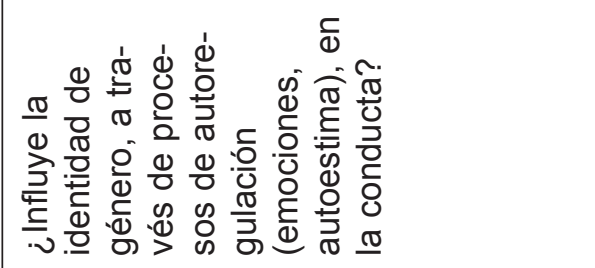 \\
\hline & 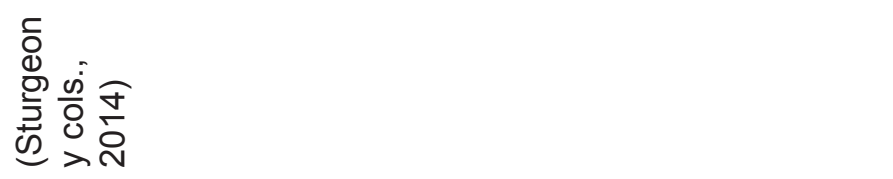 & 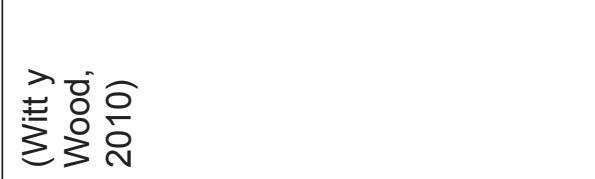 \\
\hline
\end{tabular}




\section{Referencias bibliográficas}

Aubé, J. (2008). Balancing concern for other with concern for self: links between unmitigated communion, communion, and psychological well-being. Journal of Personality, 76, 101133.

Bolger, N., Davis, A. y Rafaeli, E. (2003). Diary methods: Capturing life as it is lived. Annual Review of Psychology, 54, 579-616.

Bolger, N., DeLongis, A., Kessler, R. C. y Schilling, E. A. (1989). Effects of daily stress on negative mood. Journal of Personality and Social Psychology, 57, 808-818.

Conner, T. S. y Silvia, P. J. (2015). Creative days: A daily diary study of emotion, personality, and everyday creativity. Psychology of Aesthetics, Creativity, and the Arts, 9, 463-470.

Curran, M. A., McDaniel, B. T., Pollitt, A. M. y Totenhagen, C. J. (2015). Gender, Emotion Work, and Relationship Quality: A Daily Diary Study. Sex Roles, 73, 157-173.

Genet, J. J. y Siemer, M. (2012). Rumination moderates the effects of daily events on negative mood: results from a diary study. Emotion (Washington, D.C.), 12, 1329-1339.

Hankin, B. L., Fraley, R. C. y Abela, J. R. Z. (2005). Daily depression and cognitions about stress: evidence for a traitlike depressogenic cognitive style and the prediction of depressive symptoms in a prospective daily diary study. Journal of Personality and Social Psychology, 88, 673-685.

Kilpatrick, R. y Trew, K. (1985). Life-styles and psychological well-being among unemployed men in Northern Ireland. Journal of Occupational Psychology, 58, 207-216.

Laurenceau, J. P. y Bolger, N. (2005). Using diary methods to study marital and family processes. Journal of Family Psychology : JFP : Journal of the Division of Family Psychology of the American Psychological Association (Division 43), 19, 86-97.

Ohly, S., Sonnentag, S., Niessen, C. y Zapf, D. (2010). Diary Studies in Organizational Research. Journal of Personnel Psychology, 9, 79-93.

Reis, H. T. y Gable, S. L. (2000). Event-sampling and other methods for studying everyday experience. En H. T. Reis y C. M. Judd (eds.), Handbook of Research Methods in Social and Personality Psychology (pp. 190-222). New York, NY: Cambridge University Press.

Song, Z., Foo, M. D., Uy, M. A. y Sun, S. (2011). Unraveling the daily stress crossover between unemployed individuals and their employed spouses. The Journal of Applied Psychology, 96, 151-168.

Song, Z., Uy, M. A., Zhang, S. y Shi, K. (2009). Daily job search and psychological distress: Evidence from China. Human Relations, 62, 1171-1197.

Sturgeon, J. A., Zautra, A. J. y Okun, M. A. (2014). Associations between financial stress and interpersonal events: A daily diary study of middle-aged adults and their life circumstances. Psychology and Aging, 29, 803-813.

Witt, M. G. y Wood, W. (2010). Self-regulation of Gendered Behavior in Everyday Life. Sex Roles, 62, 635-646. 